\title{
83
}

\section{Hypermedia authoring in the classroom: but what is required to make it really creative?}

\author{
Colin Wells \\ University of Reading \\ United Kingdom
}

\begin{abstract}
Hypermedia use in schools and colleges is growing rapidly, but how much does this involve pupils in creative activities rather than simply 'using' preproduced packages? Even when engaged in authoring, how much is imaginatively creative rather than involving the selection and combination of predefined items? This paper discusses the factors involved and the necessary facilities for truly creative hypermedia authoring. This is done by identifying the range of commonly available media, and then contrasting the facilities required to use preproduced hypermedia packages with those required for authoring. Authoring facilities are further subdivided into 'passive' and 'imaginative', and the requirements for each are discussed. Finally, a few illustrations of creative hypermedia authoring are provided from the author's experience.
\end{abstract}

Main conference themes: resources, methodologies

Educational areas: primary education, secondary education, further education, higher education

Study topics:

Secondary keywords: authoring systems, creativity, hypermedia, teaching methods, tools 


\section{INTRODUCTION}

There is a potentially worrying tendency for technology to move people away from creative activities to those involving selection and manipulation of preproduced items. For example many hi-fi music systems now come with dual cassette recorders allowing recording of existing material from another component such as music CD player, radio tuner or the other cassette player, but without the provision of a microphone- or in many cases even a microphone socket-for 'live' recording. Similarly with computer systems Desk-Top Publishing (DTP) packages mainly come with a range of predefined fonts, layouts and borders, and often with no easy way to create original ones. Sound effect libraries are easily available for the creator of a sound track to select from and the prevalence of clip art is making pupils and students question the need for 'art' packages for creating illustrations for their DTP work. Similar 'libraries' are being developed for digitized photographs and video clips-thus further enticing the author of hypermedia material away from actively creating his or her own items. Of course the big attraction of using such preprepared material is speed (assuming an adequate cataloguing system), but I would maintain that the loss in relevance which this brings to the product is significant. To create fully your own picture, create and record your own sound or music, and capture your own live video for use in a computer adds tremendously to the ownership and personalizing of the material being created, and also provides a richer experience of computing and technological applications for the pupil or student concerned. While these sentiments obviously apply to many separate computer applications, these can all be considered as potential components of hypermedia authoring-which is the focus of this paper.

Hypermedia itself could be perceived in the same limited manner with pupils or students simply using preproduced material. Indeed, many so-called 'multimedia' computer systems one sees advertised are really only hypermedia playback systems - and not systems capable of allowing the creation of hypermedia material, that is not without additional hardware and software. Before discussing the contrasting levels of facilities required for play-back of hypermedia and for creative hypermedia authoring the various types of potential component media will be considered.

An obvious ingredient of hypermedia is a variety of media [1], [2]. These can be very varied and not all are required by every application (as not every feature of DTP is required in every application). 
Those available include:

- text:

range of fonts/colours/sizes, straight or in a variety of paths;

- drawings: vector graphics - defined as points/lines, monochrome/coloured;

- pictures: bitmapped images - defined as blocks of memory, monochrome/coloured;

- music:

- sound: defined notes/rhythms, variety of sounds, one or more parts/layers;

- 3D drawings: sampled sounds, music/talking/sound effects;

- 2D animation: drawings which can be moved and viewed from any angle;

- 3D animation:

- video: animated vector graphics or bit-mapped images, monochrome/coloured; animated film of 3D objects, monochrome/coloured; clips of full-motion video, with or without a soundtrack.

Fig. 1 Common types of media use in hypermedia

Just as preproduced files exist for otherwise 'content free' software such as databases, spreadsheets and programming languages, so preproduced files exist for hypermedia packages. Indeed many of these products could be looked at as a form of hypermedia database, e.g. encyclopaedia or newspaper archives. Others can appear to have more sequential features and are designed as teaching or training material, perhaps more like prewritten educational programs written in a programming language.

It could be argued that although using such types of preproduced material can be educationally beneficial, pupils or students can learn far more by creating their own material-whether in the form of a hypermedia database, training material or a presentation of research findings from an investigation (an interactive, flexible, dynamic extension of DTP presentations). Going back to the analogies mentioned above: there are parallels here with the use of spreadsheets where creating your own spreadsheet structure and using it is arguably more educationally beneficial than using prewritten spreadsheets. Or creating a small personally relevant database file can often be more beneficial than using a large prewritten file. Provided programming languages are easy and well structured people can also benefit from creating their own programs, tailor made for their own problems, rather than always relying on prewritten material.

Thus it is important to bear in mind these two styles of use: the using of preproduced material and the creation of new material. I would argue that while both are valuable, the latter has more to offer educationally. Having said this it is important to realize that these two different approaches require different facilities and a range of levels of creativity can be supported. Firstly, the facilities required for preproduced hypermedia products will be outlined. 


\section{Preproduced hypermedia}

It is a common fallacy simply to equate hypermedia with the use of CD-ROMs. Hypermedia applications do not have to be so large that CD-ROMs become the only viable method of distribution and means of access during use. Admittedly many applications such as encyclopaedia and newspaper archives mentioned above do require huge amounts of memory, but there are also a significant number of applications available on disc-mainly of educational or training material where such a medium can contain sufficient material for one or more teaching/training sessions.

Educational material of this type for schools is generally available on disc in the United Kingdom (UK) for systems such as the Acorn Archimedes (A-series) using such hypermedia programs as GENESIS or MAGPIE. (Later on the point will be made that for many creative applications-whether of teaching/training material or as a presentation of research findings-a floppy disc or at least a hard disc is often adequate for storage and distribution locally. Perhaps only a comparatively small number of people will have the time, funding and resources to create very large hypermedia applications requiring CD-ROM technology for distribution.)

In terms of inbuilt hardware some platforms are fully capable of operation for all the types of media listed above, i.e. these already have good sound facilities and are fast enough (with RISC technology) to cope with no extra hardware with replaying moving video and sound, or 3D animation. The size of files is limited only by the memory available-and thus much larger applications can be run with large hard disc systems or with the addition of a CD-ROM drive.

Some platforms do not have inbuilt sound facilities and so will need a sound board, loudspeakers and/or headphones as well as almost certainly a CD-ROM drive for commercially produced material. (This is essentially what is supplied as a 'multimedia' system.) However, with many such systems it will sometimes be necessary to purchase additional video boards (and perhaps other accelerating chips) to be able to play back moving video clips.

As mentioned above hypermedia applications require fast hardware and operating systems with particularly the following features:

- editing of pages involving several large picture files (or other large files);

- playing complex multipart music (with or without MIDI);

- recording or playing high quality sampled sounds;

- 3D drawing manipulation;

- 2D drawing and picture animation;

- 3D drawing animation;

- recording and playing moving video clips (with or without a sound track). 
Fast RISC processors are rapidly becoming necessary to cope efficiently with the size, complexity and real-time nature of many of the media in hypermedia applications. This was a major reason for Apple to move to RISC based POWER MACs even though they already had a good speed bonus through the integral operating support for sound and moving video (and the Acorn A-series systems have used RISC from the start).

\section{Creating hypermedia applications}

In terms of creating applications of hypermedia a different and much larger set of facilities is required. Furthermore, in a similar way to that discussed previously about the division of hypermedia uses into predefined or creative, the available features for creative hypermedia could be classified as passive or imaginative (or somewhere in between) depending upon whether they are inherently predefined or require some imaginative creative operations. The chart below provides a rough guide to available facilities indicating whether these are normally 'passive', 'imaginative' or somewhere in between.

\section{Passive}

(In-Between)

text

drawings clip art;

pictures clip art;

\section{Imaginative}

range of fonts/colours/sizes, straight or in a variety of paths - imported from WP or typed straight in;

new self-produced drawings; 'adjustable' clip art (i.e. allowing modification);

new pictures from art packages; video digitizer-live image capture;

video digitizer - video tape image capture; scanned picture/photograph/drawing; part/all of screen grabbed from (or produced by) an application;

music prestored tunes; original composed/arranged tunes;

sounds prestored sounds; new sampled sounds - microphone; new sampled sounds-from other device (e.g. tape rec. or music $\mathrm{CD}$ );

$3 D$ drawing prestored 3D drawings; newly created $3 \mathrm{D}$ drawings;

$2 D$ animation prestored 2D films; newly created 2D animated films;

$3 D$ animation prestored 3D films; newly created 3D animated films; video prestored video clips; newly created video clips; new captured clip from previously recorded video (with or without a sound-track).

\section{Chart 1}


Again I would make the point that in my opinion much more can be gained educationally, and much better relevant and tailor made results can be produced, through the use of the 'imaginative' side of these features rather than through the 'passive' features (e.g. clip art, prestored sounds etc.)-although one can argue that there is still the overall creative process of combining prestored elements in these cases.

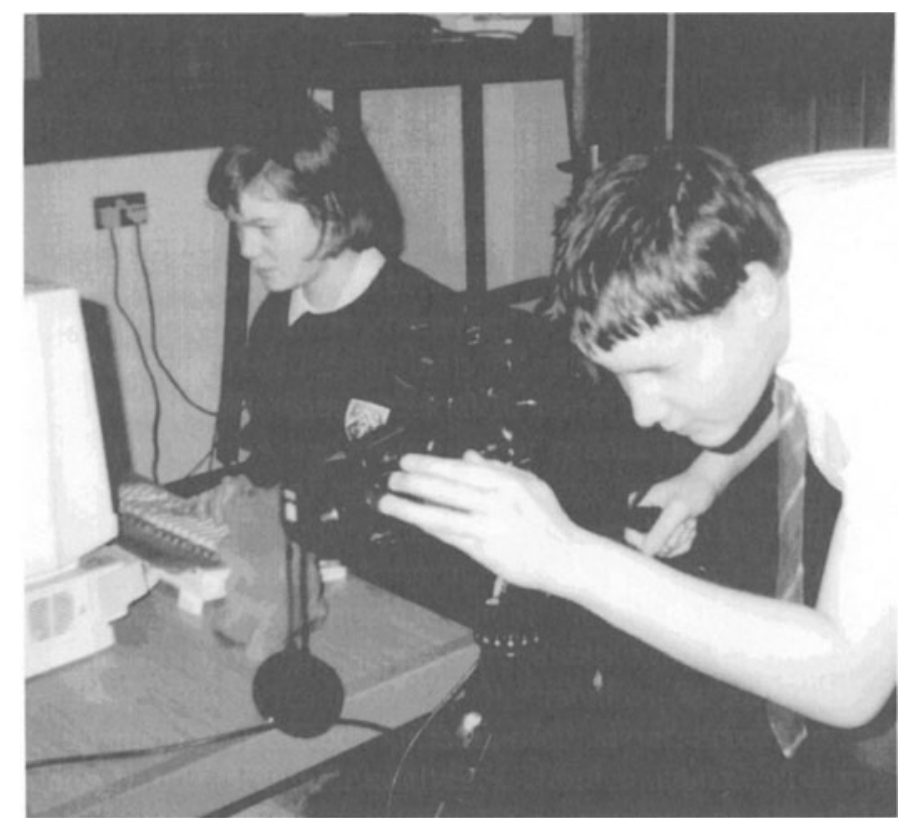

Fig. 2 Pupils using a video camera as an input to a digitizer for hypermedia authoring

The size of hypermedia files can vary considerably from a few hundred kilobytes to half a gigabyte or more depending on the purpose of the application. Just as small examples to teach the principles of programming, e.g. in BASIC or LOGO, need only be a few lines in length so too can hypermedia files created by pupils in primary or secondary schools (or students) when learning the principles of hypermedia. Applications of this type can usefully be constructed to fit onto a floppy disc (usually with some inbuilt compression routines to compact the storage). Material for teaching a simple topic, for training purposes or for presenting the results of research investigations may also fit onto a floppy disc or may require several megabytes (and therefore hard disc storage)-depending on the type of media used. 
As some measure of memory requirements the following list provides some feel for the amount of storage though of course sizes vary within each category.

low: $\quad$ text, simple defined drawings, simple music;

medium: $\quad$ complex drawings, complex music, small pictures, 2D animation, 3D drawings, short sound samples;

large: $\quad$ long sound samples, 3D animation, large pictures;

very large: video clips.

Apart from special projects, extended research and investment in the creation of suites of teaching material, hard disc storage should be sufficient for most classroom creative hypermedia activities. If much more investment in time is available to undertake a larger project or extended research to produce a very large hypermedia application, this will require a large hard disc system, e.g. a gigabyte, on which to compile the material (and hold a backup), prior to the possible creation of a finished product on CD-ROM (through a commercial firm or internally) for distribution to other users. It must be emphasized that this level of hypermedia production requires a lot of planning, developing, testing and creation time, and is not something which can be undertaken lightly. It is important to recognize that most of the time involved should be in the planning and creation of the educational ideas and framework, rather than in the physical operations of building the application.

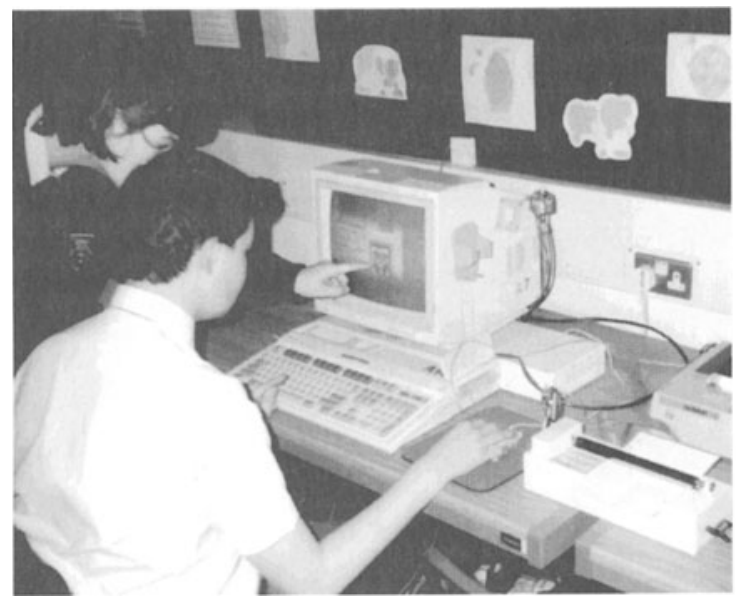

Fig. 3 Pupils scanning a school badge for use in hypermedia authoring 


\section{Extra hardware and software for creating hypermedia}

Creating hypermedia applications (rather than using preproduced applications) usually requires hardware in addition to that already mentioned as needed simply for playback. To include the range of creative media outlined in Figure 1, the following will be required:

- video digitizer board (for capture of pictures from video camera or video tape);

- scanner and interface ( for the input of own pictures, drawings, photographs etc.);

- MIDI board (to connect to music synthesiser or sound source for high quality music);

- microphone (for recording sound samples);

- leads for direct sound input from tape-recorders/music CDs or similar devices;

- video camera/recorder (for live video input or video tape applications).

Note that this is in addition to any sound and moving video boards, CD-ROM players and additional large hard disc drives already mentioned.

Similarly additional software is required for the range of creative input media listed in Figure 1:

- compatible word processing package (for importing text if required);

- compatible drawing/editing (vector graphics) program and clip art (drawings) library;

- 'adjustable' clip art program and files;

- compatible art program for picture creation/editing and clip art (picture) library;

- scanner program;

- video digitizer program;

- screen grabbing program (for whole or part of screen from any other program);

- music composing/editing program and example music file library;

- sound sampling/editing program and sound sample library;

- 3D drawing program and library;

- 2D drawing animation program and library;

- 2D picture animation program and library;

- 3D drawing animation program and library;

- moving video (and sound) capture/editing program and library.

In terms of ease of use there can be a trade-off between simplicity of operation and sophistication of the end product. For many selfproduced applications all that is required is to link together frames or pages of a variety of types which are to be activated by clicking on user defined 'hot-spots' or 'buttons' on the screen using the mouse. For such applications versions of hypermedia programs exist which work entirely using object oriented program and physical pointing operations only, i.e. not requiring any programming in any kind of written language. Such program versions are very useful in learning about 
hypermedia and for producing simple examples, presentations and teaching material.

For more sophisticated applications which may be required for larger, commercially saleable products, a script language is usually used with or without additional physical object oriented controls. A simple example of need would be if you wished a frame to open automatically ten second after another frame or button is accessed.

Not all hypermedia programs allow the creation of hypermedia applications without necessarily becoming involved in the script language-which can thus put up an additional hurdle to inexperienced people starting with hypermedia.

For some platforms hypermedia packages exist in a variety of versions which one can use entirely without accessing the script language or alternatively by using a combination of object operations and script language. Some packages require the use of a script language, and this should be checked.

\section{EXPERIENCES WITH CREATIVE HYPERMEDIA AUTHORING}

At the University of Reading Faculty of Education and Community Studies creative hypermedia facilities have existed and have been used for the last five years on the Acorn Archimedes (A-series) computer systems.

In fact all of the media listed in Figure 1 and all of the hardware and software facilities for passive and imaginative creation of hypermedia listed in Chart 1 are available, and have mostly been available for the whole five years (with moving video being added 2 years ago).

These facilities have been used for the last three years by the Master of Arts (MA) course in Information Technology (IT) in Education and by students taking the IT Module of the MA in English and Language in Education. Both of these courses provide some twenty hours of teaching concerning the authoring of hypermedia presentations including the range of component media packages and students are required to produce an example of creative hypermedia involving most, if not all, of the available range of media. This activity is seen as a valuable part of both of these courses and the students learn much more about hypermedia than they would by simply using preproduced examples. Also the encouragement to use the full range of 'imaginative' facilities significantly helps the students to personalize their material and relate to it much more effectively. In fact three MA IT in Education students have already undertaken dissertation studies concerning the creation and evaluation of hypermedia applications-one of which has involved the creation of hypermedia files by special needs primary pupils [3], [4], [5]. 
Some brief examples of 'imaginative' facilities which have been included in such applications. Students have created hypermedia presentations concerning an underwater diving trip involving the use of video pictures taken during the dive and later video digitizing of shells collected. A project on 'life cycles' by a student involved scanning of real fossils with a colour scanner and the creation of a hand drawn animated film showing the growth of a plant from a seed through its flowering to its subsequent death. A student presentation on flying included scanned photographs of light aircraft from a local airfield, the creation of 3-dimensional film animations of aircraft flying and appropriate sampled sound effects. The use of Photo Compact Disc images of a family holiday combined with text, hand drawn and scanned illustrations and originally composed music as part of a student project. A detailed project on Celtic knot design including animated sequences teaching how to create the component knots. And presentations by children involving their own voice commentaries and scanned or selfdrawn pictures of relevant personal objects such as pets or toys.

It is significant that most of these have been undertaken on 'standard' systems such as those common in UK schools-both primary and secondary-for example within 4 megabytes of main memory and often without even using hard disc storage. These experiences have demonstrated the great value of creative activities in hypermedia authoring.

As outlined above developments in hypermedia are not trivial. There is a wide variety of factors to consider. There is a need to develop adequate facilities to play back preproduced hypermedia applications, but an even greater need, particularly in an educational establishment, for good facilities for the creation of hypermedia applications-especially those facilities offering good imaginative creative support.

\section{REFERENCES}

1. British Computer Society Schools Committee (1992) Multimedia in Education. British Computer Society, London.

2. Mapp, L. (Ed.), (1993 Envision - The Multimedia Issue, NCET, Coventry.

3. Beardwood, M.E. (1992) An illustration of Hypermedia in Physical Education, unpublished dissertation for Master of Arts in IT in Education course, University of Reading. 
4. Sweatman, E.A. (1993) Early steps towards designing a hypermedia application for learning, unpublished dissertation for Master of Arts in IT in Education course, University of Reading.

5. Rickards, H. L. (1994) Investigations into certain aspects of teaching hypermedia to pupils with mild learning difficulties, unpublished dissertation for Master of Arts in IT in Education course, University of Reading. 